\title{
Research on Path Planning of Aero-Engine Blade Profile Measurement Based on Coordinate Measuring Machine
}

\author{
Kai LI ${ }^{1, *}$, Zhi HUANG ${ }^{1,2}$ and Yun HUANG ${ }^{2}$ \\ ${ }^{1}$ University of Electronic Science and Technology of China, School of Mechatronics Engineering, \\ 611731 Chengdu, China \\ ${ }^{2}$ Chongqing University, College of Mechanical Engineering, 400044 Chongqing, China \\ *457613088@qq.com
}

Keywords: Aero-engine blade, Profile inspection, Coordinate measuring machine (CMM), Path planning, Contour measurement with constant height.

\begin{abstract}
It is important to the aero-engine blade's profile inspection that the aero-engine blade's surface is highly complex with distortions. But its dimensional inspection demands a large number with measurement method most adopts the coordinate measuring machine (CMM). And the rational measurement path planning is the key to ensure the high measurement accuracy and efficiency. This paper proposed an algorithm model based on the contour measurement with constant height to solve the problem that few measuring points in the blade's leading and trailing edge will lead to distortion of measurement data.
\end{abstract}

\section{Introduction}

The blade plays a decisive role in aero-engine which is equivalent to the heart of airplane. And the blade requires extremely high processing accuracy that affects the overall performance of aeroengine. To value its quality needs using some special methods for contour measurement. Regularly detecting will find the extent of damage in the blade, used in a harsh environment, and determine whether it continues to be used. There are several difficulties on blade contour measurement: (1) Blade's high twist brings its surface complexity[1]. (2) The curvature of leading and trailing edges fast changing may lead that the measurement data is too small to restore the model. (3) Improving the measuring speed helps improving the efficiency of the measurement system, but the measurement accuracy will be reduced. (4) The repetitiveness of the measuring results reflects the accuracy of the contour measurement[2]. Replacing other measuring devices, CMM has become the main blade measurement equipment. The path planning of free-form surface measurement is the core issue in CMM. And it is the basis of profile automatic measurement, which directly affects the measurement efficiency and the precision of reconstructed surface[7].

\section{Proposed Path Planning Problem}

The profile measuring path planning mainly focus on determining the coordinates of measuring points and the measuring order of measurement path. The contour measurement with constant height in Fig. 1 is one of the most common measurement methods.

The surface data points are collected and the path planning is carried out by using the UG secondary development kit[3]. I. Ainsworth et al[4] proposed a method of extracting the measuring points by mesh surface projection. The greater the grid density, the larger the quantity of measuring points. But little points will be found in the leading and trailing edges. Lin Xiao-jun et al[2] present a blade flow path measurement method. To build the structure of the flow curve is the first step. And then, the coordinates of the sampling points and the normal vectors are calculated according to the change of curvature. 


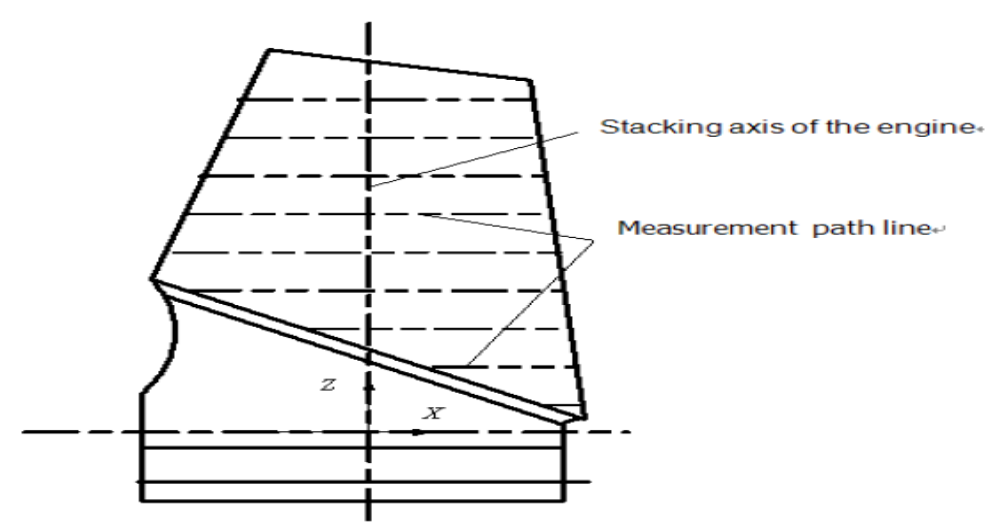

Figure 1. The Contour Measurement with Constant Height [7]

Chinese Ministry of Aviation Industry's HB5647-98 standard stipulated that the blade surface tolerances need to be controlled in a cross-sectional plane. So the contour measurement with constant height still occupies a major position. The method proposed in this paper is based on contour measurement with constant height. Firstly, make the measurement line to discrete into uniform points. Secondly, check the adjacent points' angle, if the angle between adjacent normal vectors is too large, interpolation will be performed.

\section{Path Planning Method and Realization}

\section{Path Planning Principle}

(1)The measurement path curves should cover the whole measurement area of the blade, showing uniform distribution.

(2)Every measurement path curve should be divided into four measurement areas : the section line of suction face, the section line of pressure face, the section line of leading edge and the section line of trailing edge.

(3)On the section line of leading edge and the section line of trailing edge, the curvature changes fast. The spacing between the sampling points should be relatively dense so that it is easy to show the blade surface features.

\section{Path Planning Scheme}

(1)Build parametric model of the blade surface based on theoretical section data of a blade.

(2)In accordance with the theoretical coordinate system, obtaining cross-sections lies on equidistantly intercepting the blade profile along the direction of $\mathrm{Z}$.

(3)Calculate isometric curves, the distance between two isometric curves is the radius of probe head.

(4)Discretize the isometric curve and use projection method to calculate measuring points and normal vectors.

(5)Interpolate values between adjacent points, which have a large normal vector angle.

(6)Sort the measuring points and store them.

\section{Algorithm Model}

\section{Isometric Curve Model}

CAI's[5] algorithm of generating the isometric curve for the NURBS curve is adopted in this paper. The non-uniform rational B-splines (NURBS) curve is used to represent the blade cross-section profile:

$$
C(u)=\frac{\sum_{i=0}^{n} w_{i} d_{i} N_{i k}(u)}{\sum_{i=0}^{n} w_{i} N_{i k}(u)}, u \in U
$$


Where $d_{i}(i=0,1, \ldots, n)$ stands for the controlling vertex, $w_{i}$ stands for the weighted factor, $n$ stands for the number of controlling vertexes, $N_{i k}(u)$ stands for K-th B-spine basis functions, $N_{i k}(u)$ can be expressed by the cox-de boor algorithm as:

$$
\left\{\begin{array}{c}
N_{i, 0}(u)=\left\{\begin{array}{c}
1, u_{i} \leq u \leq u_{i+1} \\
0, \text { else }
\end{array}\right. \\
N_{i, k}(u)=\frac{u-u_{i}}{u_{i+k}-u_{i}} N_{i, k-1}(u)+\frac{u_{i+k+1}-u}{u_{i+k+1}-u_{k+1}} N_{i+1, k-1}(u)
\end{array}\right.
$$

$u \in U, U=\left\{0, \ldots, 0, u_{k+1}, \ldots, u_{n}, 1, \ldots 1\right\}$, Calculate the parametric chord length as follow:

$$
\left\{\begin{array}{c}
l_{i}=\left|d_{i}-d_{i-1}\right| \\
u_{i}-u_{i-1}=\frac{\sum_{j=i-k}^{i-1} l_{j}}{\sum_{i=k+1}^{n+1} \sum_{j=i-k}^{i-1} l_{j}}
\end{array}\right.
$$

$i=k+1, k+2, \ldots, n+1$. If $C(u)=(x(u), y(u))$, the isometric curve could be expressed as:

$$
\begin{aligned}
& D(u)=C(u) \pm d \times N(u) \\
& =C(u) \pm d \times \frac{\left(-y^{\prime}(u), x^{\prime}(u)\right)}{\sqrt{x^{\prime}(u)^{2}+y^{\prime}(u)^{2}}}
\end{aligned}
$$

$N(u)$ is the unit normal vector of the parameter $u$ for $C(u)$, so $N(u)=\left(x^{\prime}(u), y^{\prime}(u)\right)$. The key is to derive the B-spline basis functions. Calculate the derivative of equations(2):

$$
\left\{\begin{array}{c}
N_{i, 0}^{\prime}(u)=0 \\
N_{i, k}^{\prime}(u)=\frac{1}{u_{i+k}-u_{i}} N_{i, k-1}(u)+\frac{u-u_{i}}{u_{i+k}-u_{i}} N_{i, k-1}^{\prime}(u) \\
-\frac{1}{u_{i+k+1}-u_{k+1}} N_{i+1, k-1}(u)+\frac{u_{i+k+1}-u}{u_{i+k+1}-u_{k+1}} N_{i+1, k-1}(u)
\end{array}\right.
$$

By solving equations (1), (2), (3), (4) and (5), an isometric curve of the NURBS curve can be generated.

\section{Curve Discretization}

Discrete data are required to be uniformly distributed over the blade cross-section line. The algorithm is based on equal arc-length algorithm of Chun-yang JIA[6]. Firstly, the length of the curve is calculated :

$$
\begin{gathered}
L=\sum_{p=0}^{n-1} H_{p} \\
=\sum_{p=0}^{n-1} \sum_{u_{p}}^{u_{p+1}} \sqrt{\left[D_{x}^{\prime}(u)\right]^{2}+\left[D_{y}^{\prime}(u)\right]^{2}+\left[D_{z}^{\prime}(u)\right]^{2}} d u \\
=\int_{0}^{1} \sqrt{\left[D_{x}^{\prime}(u)\right]^{2}+\left[D_{y}^{\prime}(u)\right]^{2}+\left[D_{z}^{\prime}(u)\right]^{2}} d u
\end{gathered}
$$

Where $H_{p}$ is corresponding to the NURBS curve segment arc length in domain $\left[u_{p}, u_{p+1}\right)$. Define an arc length step size quadratic function as:

$$
L=L_{a}(s)=a \times s^{2}+b \times s+c
$$

The accuracy of the discrete curve is changed by adjusting the coefficients $a, b, c$ and the step parameter $s$. Calculate discrete segment number as: 


$$
N^{\prime}=\frac{L}{L_{a}(s)}
$$

Round the result to get discrete segment number $N . \sum_{p=0}^{m} H_{p}$ is corresponding to the NURBS curve segment arc length in domain $\left[0, u_{m+1}\right)$. And the total length is equal $L_{n}=n L_{a}(s)$. Here is an equation could Indicate the location of the discrete point:

$$
\begin{aligned}
& \int_{u_{m+1}}^{u} \sqrt{\left[D_{x}^{\prime}(u)\right]^{2}+\left[D_{y}^{\prime}(u)\right]^{2}+\left[D_{z}^{\prime}(u)\right]^{2}} d u \\
& =L_{n}-\sum_{p=0}^{m} H_{p}
\end{aligned}
$$

If $u \in\left[u_{m}, u_{m+1}\right)$, the equation (9) should be $L_{n}-\sum_{p=0}^{m} H_{p}<0$. So parameter $u$ can be found, then substitute it into equation (1) to obtain the discrete point.

\section{Projection Model}

The minimum distance of a discrete point $A\left(x_{0}, y_{0}, z_{0}\right)$ to a point $B(x(u), y(u), z(u))$ on the section curve can be expressed as:

$$
\begin{aligned}
& d \operatorname{di}_{\text {min }}=\min [|A B|] \\
& =\min \left[\sqrt{\left[x_{0}-x(u)\right]^{2}+\left[y_{0}-y(u)\right]^{2}+\left[z_{0}-z(u)\right]^{2}}\right]
\end{aligned}
$$

Calculate the parameter corresponding shortest distance by iteration using the selected step size. Then substitute the parameter into equation (1) to obtain the projection points.

\section{Interpolation}

According to the calculated projection points and the discrete points, the normal vectors of the discrete points can be computed. If the adjacent normal vectors are $\left(\alpha_{1}, \beta_{1}, \gamma_{1}\right)$ and $\left(\alpha_{2}, \beta_{2}, \gamma_{2}\right)$, the angle between two normal vectors could be expressed as:

$$
\cos \theta=\frac{\left|\alpha_{1} \alpha_{2}+\beta_{1} \beta_{2}\right|}{\sqrt{\alpha_{1}^{2}+\beta_{1}^{2}} \sqrt{\alpha_{2}^{2}+\beta_{2}^{2}}}
$$

Set a upper limit value $\varepsilon$. If $\cos \theta<\varepsilon$, it means that the angle is too large. It has to take the midpoint of the adjacent points for interpolation. And use the shortest distance projection method to calculate the projection point.

\section{Program Flow Diagram}

The measuring path would be as Fig.2 shows.And according to the above formulas and the designed steps, complete the program as shown in Fig.3.

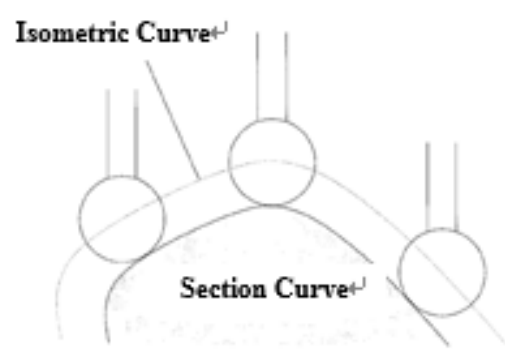

Figure 2. Measuring Path[4] 


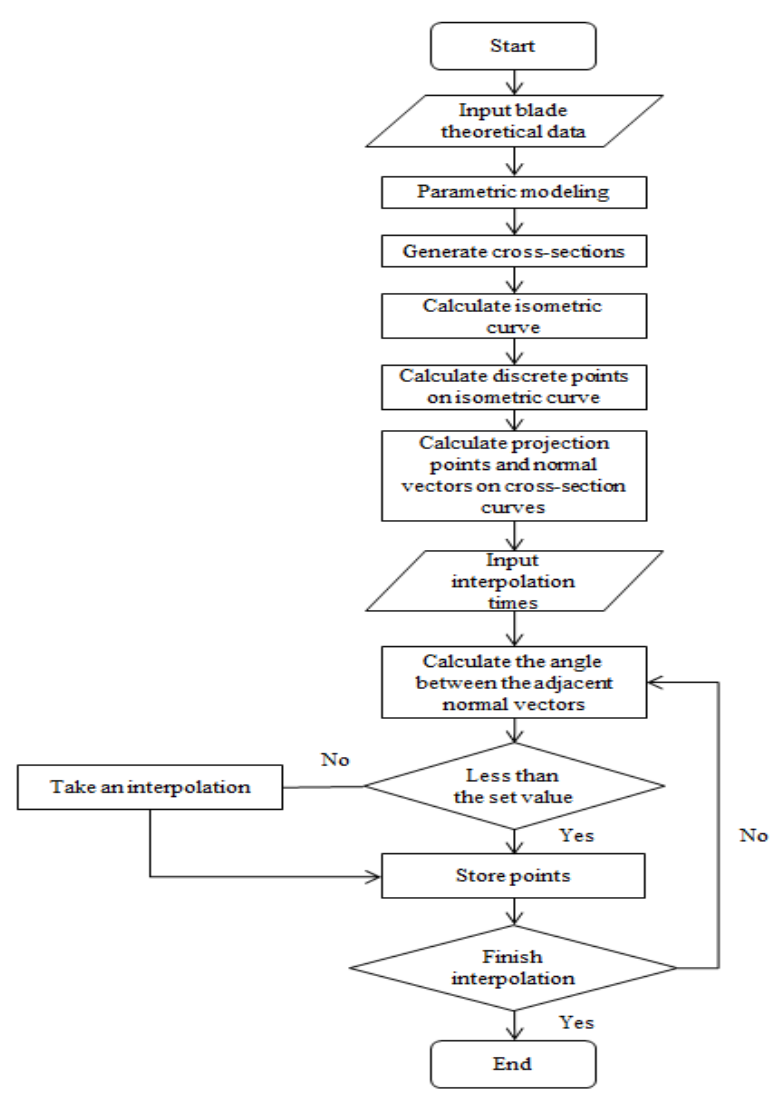

Figure 3. Progaram Flow Chart

\section{Verification}

A model of a blade was used to validate proposed method. Using 8 planes with constant height layered it into cross-sections as shown in Fig.4, and 8 cross-sectional profiles were obtained.

Taken at $1 \mathrm{~mm}$ arc length spacing, discrete measuring points were uniformly distributed on crosssectional profiles. And then the projection was calculated. The result is shown in Fig.5.

Figure 4. Cross-section Curves

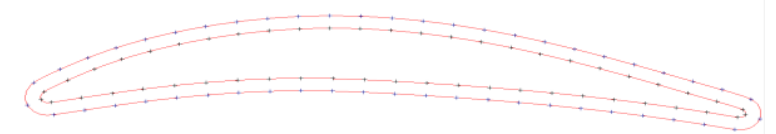

Figure 5. Discrete Points and Projection Points

Then calculate the angle between the adjacent normal vectors. Set $\arccos (\varepsilon)=3^{\circ}$. If the angle more than $3^{\circ}$, interpolation is necessary. After interpolation, final measuring sampling points were determined which is shown in Fig.6 and Fig.7. 


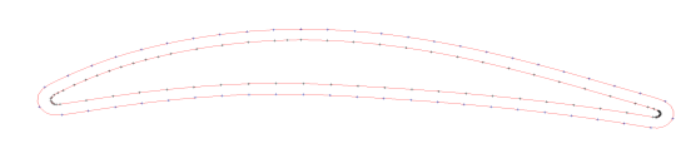

Figure 6. The Points after Interpolation

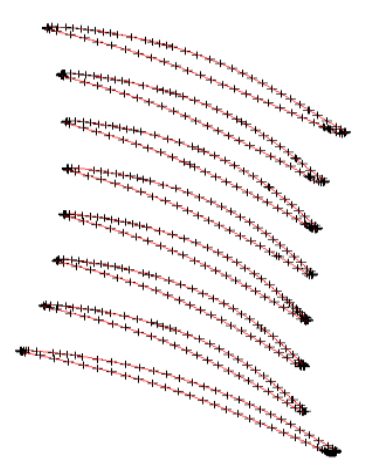

Figure 7. Final Sampling Points

\section{Summary}

On account of the curvature of leading and trailing edges on cross-sectional profiles changes fast, less of measuring point will not be able to truly restore the size information of the blade surface and reflect effectiveness of measuring data. The method proposed in this paper is suitable for contour measurement constant height, which is possible to obtain a more intensive set of measurement sample points and helpful for data processing by adjusting the interpolation times and the angle upper limit value.

\section{Acknowledgement}

This paper is supported by the Fundamental Research Funds for the Central Universities (No.ZYGX2015J086).

\section{References}

1. X. YU, P. YE. Aero - engine Compressor Blade Profile Detection Technology[J]. Aeronautical Manufacturing Technology, 2007(11):48-50(in Chinese).

2. X. LIN, C. SHAN, Z. WANG, Y. SHI. Measurement Techniques of Coordinate Measuring Machine for Blade Surface of Aero-engine[J]. Computer Integrated Manufacturing System, 2012, 18(1):125-131(in Chinese).

3. W. MA. Study on Extracting Characteristic Parameters of Blade Section of Aeroengine[D]. Tianjin :Tianjin University, 2013(in Chinese).

4. I. Ainsworth, M. Ristic, D. Brujic. CAD-Based Measurement Path Planning for Free-Form Shapes Using Contact Probes[J]. The International Journal of Advanced Manufacturing Technology, 2000, 16(1):23-31.

5. T. CAI, Y. ZHAO, Z. WANG, et al. The Generation Algorithm of Planar NURBS Curve and Its Offset[J]. Machinery Design \& Manufacture, 2014(7):224-227(in Chinese).

6. C. JIA, Y. YANG, F. CHEN, et al. NURBS curve discrete algorithm based on equal arc-length principle. Computer Engineering and Applications[J], 2014, 50(3): 165-167(in Chinese).

7. X. LIN, S. JIANG, X. LIU, et al. The CMM Measurement Path Planning for Blade Surface Based on the Contour Measurement[C]// Second International Conference on Digital Manufacturing and Automation. IEEE, 2011:1228-1232. 